\title{
25 Research Suare \\ Inter-Cluster Competition and Resource Partitioning \\ May Govern the Ecology of Frankia
}

Indrani Sarkat

University of North Bengal

Gargi Sen

University of North Bengal

S Bhattacharyya

University of North Bengal

Maher Gtari

University of Carthage

Arnab Sen ( $\sim$ senarnab_nbu@hotmail.com )

University of North Bengal https://orcid.org/0000-0002-2079-5117

\section{Research Article}

Keywords: Reverse ecology, R, Resource partitioning, Frankia, Competition index, Complementation index

Posted Date: December 22nd, 2021

DOI: https://doi.org/10.21203/rs.3.rs-1183956/v1

License: (9) This work is licensed under a Creative Commons Attribution 4.0 International License.

Read Full License 


\section{Abstract}

Microbes live in a complex communal ecosystem. The structural complexity of microbial community reflects diversity, functionality as well as habitat type. Delineation of ecologically important microbial populations along with exploration of their roles in environmental adaptation or host-microbe interaction has a crucial role in modern microbiology. In this scenario, reverse ecology (the use of genomics to study ecology) plays a pivotal role. Some studies have reported the presence of other non-Frankia genus from the same root nodule from where the Frankia was isolated. Since co-existance of two different genus in one small niche should maintain a strict direct interaction, it will be interesting to utilize the concept of reverse ecology in this scenario.

Here, we exploited an ' $\mathrm{R}$ ' package, the RevEcoR, to resolve the issue of co-existing microbes which are proven to be a crucial tool for identifying the nature of their relationship (competition or complementation) persisting among them. Our target organism here is Frankia, a nitrogen-fixing actinobacterium popular for its genetic and host specificity nature.

According to their plant host, Frankia has already been subdivided into four clusters $\mathrm{Cl}, \mathrm{Cll}, \mathrm{CIII}$ and $\mathrm{CIV}$. Our results revealed a strong competing nature of $\mathrm{Cl}$ Frankia. The competition index between $\mathrm{Cl}$ and $\mathrm{CIII}$ was greater than other studied Frankia clusters. The other interesting result was the co-occurrence of C-II and C-IV groups. It was revealed that these two groups follow the theory of resource partitioning in their lifestyle. Metabolic analysis along with their differential transporter machinery validated our hypothesis of resource partitioning among C-II and C-IV group.

\section{Introduction}

The term adaptation is often considered as the cornerstone of ecology and evolutionary study (Hugenholtz et al. 1998). Assorted traits allowing an organism to survive in a specific habitat as well as shaping the interface between environment and genome help them to endure natural selection pressure (Hugenholtz et al. 1998). Broadly, any living organism has to contend with several different forces namely, intra-species competition, inter-species competition, natural selection as well as cooperation synergy like mutualism, commensalism etc. which implies beneficial effects on one group on another group of organism. Permanent residents of a niche are thus, the end product or "entangled bank" of all three selective forces (Gilbert et al. 2009). Nevertheless, there exists a dauntingly complex relationship among the myriad co-inhabiting strains in each niche. Deciphering this relationship is an extremely challenging task since it includes different environmental and genomic variations. Ecological dynamics and communities are potentially driven by the interactive forces among occupied species (Berlow et al. 2009). That is why the term "species interaction network" is of paramount importance in community structure study. So far our knowledge goes; the ecological network is a mathematical model representing the concept of interaction between two species (Dunne 2006). Conversely, recent advancements in computational approaches entitled us to more specialized models to fine-tune the previous ones (Poisot et al. 2015). These new lines are better in quantifying the statistical and numerical properties of 
interaction networks enhancing their robustness (Dunne 2002), productivity and durability (Duffy et al. 2007). Ings et al. (2009) mentioned that these methods were mainly focused on the food web, nevertheless, they can be applied successfully to all types of biological and more specifically ecological interactions (Kéfi et al. 2012). Communication and metabolic networks are scale-free networks where the structure and evolution are connected or integral (Zhang 2011). The major advantage of this type of network is its non-static nature i.e. these networks constantly change on the arrival of new nodes and links (Barabasi and Albert 1999, Barabasi 2009). Some researchers have also pointed out that; ecological interaction network is rather distinct from that of ecological association networks (Suzuki et al 2017). They have grouped the co-occurrence network (Faust and Raes 2012) as well as the correlation network (Friedman and Alm, 2012) as ecological association networks (Berry and Widder 2014). Biological research has been enriched with co-occurrence and co-abundance studies. Researchers have also focused on the bacterial world with the aforementioned point of view and excavated its potency in studying complex microbial ecosystems (Berry and Widder 2014, Lassalle et al. 2015). The connection between genomics and ecology can be established only by considering different aspects of ecological relationships including both mutualism and competition and correlating these interactions with niche preference of respective organisms (Lassalle et al. 2015).

However, a comprehensive study on ecological genomics needs a thorough understanding of genomic machinery regulating the response of a species to an environmental clue (Levy and Borenstein 2012). The traditional approach was to identify an ecologically adaptive phenotype first and detect associated genetic variations (Amann et al 1995). However, with the amelioration of functional genomics and system biology, a new archetype is popularly known as Reverse Ecology has been established. This method conjectures the ecology of an organism from its genomic feature (Levy and Borenstein 2012). Reverse Ecology especially emphasizes microbial ecology. Population genomics is also an example of a reverse ecology concept focusing on the complex biological system (Borenstein et al. 2008). Several tools like NetSeed (Carr et al. 2012) and NetCorporate (Levy et al. 2015) were developed for understanding the interaction among species and their environment. Nevertheless, they did not support the metabolic reconstruction of organisms and were also limited to small-scale analysis (Cao et al 2016). Much of these problems have been taken care of by a recently developed R-based program called RevEcoR (Cao et al 2016). RevEcoR utilized the reveres ecological framework for reconstructing the metabolic network of an organism. Here species interaction can be calculated by two means: competition and complementation.

Frankia is well known for its nitrogen-fixing ability while remaining associated with actinorhizal plants. Mainly three groups of actinorhizal plants are reported to be associated with Frankia and they areFagales (Betulaceae, Casuarinaceae, Myricaceae), Cucurbitales (Datiscaceae, Coriariaceae) as well as Rosales (Rosaceae, Elaeagnaceae, Rhamnaceae) (Sellstedt, A. and Richau 2013, Berry et al. 2011). They are pioneer plants in early successional sites and disturbed sites hence, are have a pivotal impact on late successions. Frankia contributes to the fitness of their host plants by providing nitrogenous compounds (Sellstedt, A. and Richau 2013). 
This symbiosis has been found worldwide with a broad range of ecological and environmental conditions mainly in poor and trivial-productive soils (Schwencke and Carú, 2001; Benson and Dawson, 2007; Sen et al. 2014; Simonet et al 1986) and helps in early plant succession. Diversity also exists among Frankia as well as their host plants. Approximately 288 species of 24 genera belonging to 8 families of 7 orders have been reported to make a symbiotic association with Frankia (Cronquist 1968, Schwintzer 2012) (please refer to Supplementary figure 1 for the taxonomic information of Actinorhizal plants). The phylogeny of Frankia distributed them into four distinct clades chiefly based on their association with host plants. Clade (C)-I, II and III contained nodulating nitrogen fixers Members of C-I can infect Myricaceae, Betulaceae, and Casuarinaceae families (except Gymnostoma). Coriariaceae, Dryadoideae (all actinorhizal Rosaceae), Datiscaceae, and Ceanothus (Rhamnaceae) are infected by C-II Frankia. Most promiscuous C-III Frankia associates with Colletieae (all actinorhizal Rhamnaceae except Ceanothus), Elaeagnaceae, Myricaceae, Casuarinaceae (only Gynmmostoma) and occasionally Alnus (Normand et al. 2007). While the fourth Clade (C-IV) possessed "atypical" strains that are either unable to re-infect actinorhizal host plants or are impotent of fixing N2 in the nodules (Normand et al. 2007). Interestingly, the ineffective C-IV strains have been isolated from Ceanothus root nodules along with C-III Frankia and both were present on the outer cover of the nodules. Thus, this phylogeny arises four distinct questions about Frankia host specificity and categorically to Frankia ecology. However, there are still some missing links that exist in the host specificity nature of Frankia. For instance,

a.) How C-I and C-III Frankia are sharing the same family of host plants?

b.) Is it possible that C-II and C-IV share the same root nodule or at least the same location of a root nodule together?

c.) Why Ceanthous can only be infected with C-II and not by C-III members?

d.) Why Gynmmostoma can only be infected with C-III and not by C-I Frankia?

To explore the possible explication of these four queries we have taken up an in-silico approach which will virtually throw light on the ecological aspect of Frankia.

\section{Material And Methods}

\section{Genome selection and sequence retrieval}

A total of 44 Frankia genomes whose complete genome sequences are available in the IMG database were selected for this study (Supplementary Table 1). The protein-coding gene sequences and translated amino acid sequences of select strains were downloaded from the IMG database (https://img.jgi.doe.gov/). A whole genome-based phylogenetic tree was generated for getting a clear view of the clustering pattern of considered Frankia. A whole genome-based phylogeny tree was generated through Type (Strain) Genome Server (TYGS) (https://tygs.dsmz.de) (Meier-Kolthoff and Göker 2019). TYGS sub-divided the whole genome-based phylogeny generation into a pair-wise comparison of 
genome sequences using Genome Blast Distance Phylogeny (GBDP) and accurate inter-genomic distances calculation by 'trimming' and distance formula (Meier-Kolthoff et al. 2013). 100 distance replicates were used for that analysis. Digital DNA-DNA hybridization (DDH) scores and confidence intervals were calculated through GGDC2.1 server (Meier-Kolthoff and Göker 2019). Intergenomic distance-based phylogeny was generated through a balanced minimum evolution tree with branch support via FASTME 2.1.6.1 (Lefort et al. 2015). Branch point support was inferred from 100 pseudobootstrap replicates per branch. The phylogeny was generated using the default parameters. Heatmaps with species cluster, sub-species cluster, $\mathrm{G}+\mathrm{C}$ amount, delta statistics, the genome size (bp) and protein content were generated through the TYGS server using default parameters.

\section{Metabolic pathway prediction}

All the strains included in this study are still not there in the KEGG database (Kanehisa 2002). Hence, to keep the uniformity, we went for predicting the metabolic pathways for all the studied strains. KEGG Automatic Annotation Server (KAAS) (https://www.genome.jp/tools/kaas/) (Moriya et al 2005) was used for this prediction and annotation. This tool gave us detailed information about the KO assignments along with substrates and products of metabolic reactions. This information was used for further analysis. BBH (bi-directional best hit) assignment method was used for this analysis. Metabolic pathways of Frankia inefficax (fri), Frankia sp. EAN1pec (fre), Frankia casuarinae (fra), Frankia alni (fal) (as assigned by KEGG) were used as a database for KO profiling of other Frankia genomes. We compared the results of KAAS and KEGG database and found them to be exactly the same thus validating our aforementioned approach. MetaCyc server (Caspi et al. 2007) was used to compare the metabolic pathway profiling among different clusters of Frankia.

\section{Reverse Ecology analysis}

Reverse Ecology analysis was done using the RevEcoR program (Cao et al. 2016) on R-studio (Allaire 2012). This analysis takes account of all the metabolic network data available for a single genome and compares it with other studied genomes. Based on the resources used by the studied organism a competition and complementation value is calculated. Reverse ecology analysis on investigated Frankia genomes assessed an overall interaction in terms of both competition and complementation among members of this genus. First we obtained the KEGG Ontology (KO) profiles of all considered Frankia. Those KO profiles were fed into RevEcoR program in R. Seed sets were generated with the command getSeedSets and seed.set. Finally, competition and cooperation index were calculated with the "complementarity index(net[[1]],net[[2]], seed.sets[[1]], seed.sets[[2]])" and "competition index(net[[1]],net[[2]], seed.sets[[1]], seed.sets[[2]])" The competition and complementation indices were generated using default parameters in RevEcoR. The result was visualized by heatmaps. Heatmaps were generated with these data in $\mathrm{R}$ for better visualization and easy understanding.

\section{Results \& Discussion}

\section{Competing behavior of Clad-I (C-I) Frankia}


The whole genome-based phylogeny divided the Frankia genus into four distinct groups (Supplementary figure 2). The major group was C-I with 23 strains and the smallest group was C-II with four strains. C-III and C-IV contained 11 and 6 strains respectively. Results obtained from reverse ecology analysis were analyzed as per the categorization obtained from this whole genome-based phylogeny. It was found that competition was more among different Frankia clusters than complementation or mutualism. Intracluster mutualism was greater than inter-cluster mutualism (Supplementary file 1).

The reverse ecology analysis established C-I Frankia as a general adversary against all other clusters especially C-III and C-IV (Supplementary file 1). Substantially high competition value with low complementation index was seen among $\mathrm{C}-\mathrm{I}$ and III indicating their antagonistic nature. Both these clusters share host plants (Myricaceae and Casuarinaceae). It is well expected that there will be a tight competition between these two clusters when they infect the Myricaceae group of plants. It was reported earlier that, C-I can infect the members from the Casuarinaceae especially Casuarina and Allocasuarina, however Gynmmostoma which is also from Casuarinaceae is infected only by C-III Frankia (Normand et al. 2007, Ngom et al. 2016). This result completely supports our finding of opposing or competing for behavior between $\mathrm{C}-\mathrm{I}$ and $\mathrm{C}-\mathrm{III}$. Thus, the high competition between these two clusters has resulted in host-specificity among them. Competition between C-I and C-III was evident from the high competition and low complementation index between them. The complementation index between $\mathrm{C}$-I and C-III varied from 0.00 to 0.56 , whereas the competition index ranged from 0.55-0.99.

Some cluster III Frankia strains like R43 (Pujic et al. 2015), Ccl149 (Mansour et al. 2017) and G2 (Nouioui et al. 2016) were indeed isolated from Casuarina root nodules however they were not grouped into the Cluster-I group because they were incapable of infecting the Casuarina roots but were capable of infecting the Elaeagnaceae group. Based on that, they were confirmed to be Cluster III Frankia. Vemulapally et al. 2019 (Vemulapally et al. 2019) worked on R43 and confirmed that this strain was isolated from Casuarina nodules as a surface contaminant. Similarly, Ccl149 and G2 are also ineffective towards Casuarina and Alnus but can infect Elaeagnus and Hippophäe Mansour et al. 2017, Nouioui et al. 2016). Their spore physiology, pigment patterns are different from C-I Frankia strains (Mansour et al. 2017). Although detailed experimental analysis has not been done on Ccl149 and G2, there is a high probability that those two strains were also isolated from Casuarina root nodule as a result of surface contamination as well. The reverse ecology analysis also showed distinct competition and less complementation between C-I and C-III clusters which supports the experimental results of Vemulapally et al. 2019 (Vemulapally et al. 2019) indicating less mutualism and more competition among C-I and C-III Frankia.

This result supports the fact of host differentiation between these two groups. Similar antagonistic nature was found between $\mathrm{C}-\mathrm{I}$ and $\mathrm{C}-\mathrm{Il}$ supporting their host differentiation nature. Overall, C-I Frankia was a common competitor against the other three clusters. It is possible that due to this competition, C-I Frankia remains restricted to the Hamamelidae subclass of actinorhizal plants and cannot infect other potent subclasses (Normand et al. 2007; Ben et al. 2018) i.e., Rosidae, Dilleniidae and Magnoliidae which are the main host plants for C-II, C-III and C-IV. 


\section{C-III members infect C-I specific host plant family due to shared metabolic pathways}

It has been reported in previous studies that, C-III Frankia can infect Eleagnace-ae, Rhamnaceae, Myricaceae, Gynmmostoma (Casuarinaceae), and sometimes Alnus. Among them, Alnus and Myricaceae can also be infected by C-I Frankia (Normand et al. 2007, Ngom et al. 2016, Benson et al. 2004). However, reverse ecology analysis revealed strong competition between them. We found high competition index value among C-I and C-III Frankia (Supplementary File 1- sheet name 'competition_Cl_CIII') and a low complementation value among them (Supplementary File 1- sheet name 'complementation_Cl_CIII'). The competition index between $\mathrm{Cl}$ and $\mathrm{C}$-III Frankia ranged from 0.55 to 0.99 whereas the complementation was quite low (0.00-0.5 approximately). A similar metabolic strategy may be one of the reasons for a similar host association. A comparative study on metabolic pathways revealed 213 shared (common) pathways between these two aforementioned groups (Supplementary file 2). This indicates that both C-I and C-III Frankia use the same nutrients for nodulation and nitrogen fixation. Moreover, a study on the nodulation signaling of Frankia proposed the presence of C-I specific NIN factor (nodulation inducing factor) among C-III members (38) which supports our hypothesis that both C-I and C-III use similar resources as well as an identical signaling cascade (at least to some extent) which resulted in the fact that, both C-III and C-I can infect Myrica and Alnus.

\section{Resource partitioning among C-II and C-IV strains}

Previous studies have reported the isolation of C-II and C-IV strains from a single nodule (Amann et al. 1995). However, our present reverse ecology analysis revealed more competition and less complementation between these two groups. From the competition index (Supplementary figure 3a, 3b) among C-II and C-IV stains, we see that the intra-cluster competition among Cll members ranged from 0.35 to 0.48 and for C-IV the values ranged from 0.31-0.49. However, the inter-cluster competition between $\mathrm{C}-\mathrm{Il}$ and $\mathrm{C}-\mathrm{IV}$ was higher than the intracluster competition (0.69-0.87). Hence a distinct competition between $\mathrm{C}-\mathrm{II}$ and $\mathrm{C}-\mathrm{IV}$ was evident. Moreover, when we compared the complementation index of C-II and C-IV strains, inter-cluster complementation was more than inter-cluster complementation. These raise the question that, how two excessively competing groups can share the same nodule at a time and grow successfully in them?

To understand the ecological aspect lying behind this highly fascinating result, we looked at comprehensive metabolic pathways of those clusters (C-II and C-IV) (Table 1; comparative metabolic pathways among four Frankia cluster provided in Supplementary file 2). Most of the metabolic pathways showed a difference between C-II and C-IV strains, however, the major disparity was observed in aromatic amino acids metabolism and carbohydrate metabolism (Table 1). It's a well-established fact that both aromatic amino acids and carbohydrates are crucial aspects of nodulation and nitrogen fixation being the major source of carbon and nitrogen (Borenstein et al. 2008; Carr et al 2012). A discrepancy of metabolism among C-II and C-IV strains could be a possible reason for the fact that these strains share the same host and same nodules irrespective of their high contest since they utilize different sources of 
energy. This provides a paradigm for a vital concept of ecology termed niche partitioning or more specifically resource partitioning (Levy et al. 2015) which states that competing species can co-exist by using the same environment differently. Moreover, when we compared the transporter families among C-II and C-IV Frankia (Fig. 1), it revealed that prominent differences. Only $1.5 \%$ of genes were shared among C-II and C-IV Frankia strains. This further validates our hypothesis of resource partitioning between C-II and C-IV. More interestingly, there were very few similarities among the four clusters of Frankia. Only 8.2\% of transporters (Fig. 1) are shared among all four Frankia clusters which support their host-specific nature largely. 
Table 1

Comparative account of metabolic pathways between C-II and C-IV Frankia strains. X indicates the presence of the specific metabolic pathway.

\section{Pathway Class}

Biosynthesis - Amino Acid Biosynthesis

$\beta$-alanine biosynthesis III

L-asparagine biosynthesis I

L-asparagine biosynthesis II

L-cysteine biosynthesis I

L-cysteine biosynthesis III (from L-homocysteine)

L-ornithine biosynthesis I
C-IV C-II

$\mathrm{X}$

$X$

$X$

$\mathrm{X}$

\section{Biosynthesis - Carbohydrate Biosynthesis}

dTDP-4-O-demethyl- $\beta$-L-noviose biosynthesis

heptadecane biosynthesis

$\mathrm{X}$

Biosynthesis - Cofactor, Prosthetic Group, Electron Carrier, and Vitamin Biosynthesis

1,4-dihydroxy-2-naphthoate biosynthesis

N10-formyl-tetrahydrofolate biosynthesis

$X$

biotin biosynthesis from 8-amino-7-oxononanoate I

$\mathrm{X}$

factor 420 polyglutamylation

$X$

folate transformations I

$\mathrm{X}$

heptaprenyl diphosphate biosynthesis

$\mathrm{X}$

NAD biosynthesis from 2-amino-3-carboxymuconate semialdehyde

$\mathrm{X}$

tetrahydrofolate salvage from 5,10-methenyltetrahydrofolate

$x$

thiazole biosynthesis II (aerobic bacteria)

$\mathrm{X}$

ubiquinol-8 biosynthesis (prokaryotic)

$X$

\section{Biosynthesis - Fatty Acid and Lipid Biosynthesis}

cis-vaccenate biosynthesis

$X$

CDP-diacylglycerol biosynthesis II

$\mathrm{X}$

phosphatidylethanolamine biosynthesis I

$X$

Biosynthesis - Metabolic Regulator Biosynthesis 


\section{Pathway Class}

glucosylglycerate biosynthesis I $\quad \mathrm{X}$

mannosylglucosylglycerate biosynthesis I $\quad \mathrm{X}$

\section{Biosynthesis - Other Biosynthesis}

L-dopachrome biosynthesis

Degradation/Utilization/Assimilation - Amine and Polyamine Degradation

4-aminobutanoate degradation II

4-aminobutanoate degradation III

$\mathrm{N}$-acetylglucosamine degradation I

creatinine degradation I

urea degradation I $x$

$x$

\section{Degradation/Utilization/Assimilation - Aromatic Compound Degradation}

2,2'-dihydroxybiphenyl degradation

2-chlorobenzoate degradation

4,5-dichlorocatechol degradation

$X$

4-chloronitrobenzene degradation

$\mathrm{X}$

4-nitrotoluene degradation II

$\mathrm{X}$

benzene degradation

$\mathrm{X}$

biphenyl degradation $\mathrm{X}$

carbazole degradation $\mathrm{X}$

chlorinated phenols degradation

cinnamate and 3-hydroxycinnamate degradation to 2-oxopent-4-enoate

$X$

diphenyl ethers degradation $X$

gentisate degradation I $\mathrm{X}$

phenol degradation I (aerobic)

protocatechuate degradation II (ortho-cleavage pathway)

urate conversion to allantoin I

$x \quad x$

\section{Degradation/Utilization/Assimilation - Carbohydrate Degradation}




\section{Pathway Class}

D-galactose degradation I (Leloir pathway) $\quad$ X

D-galactose degradation V (Leloir pathway) $\quad X$

fructose degradation $\quad x$

$\begin{array}{lc}\text { kojibiose degradation } & X\end{array}$

L-arabinose degradation I $\quad$ X

L-rhamnose degradation I $\quad X$

melibiose degradation $\quad x$

xylose degradation I $\quad X$

Degradation/Utilization/Assimilation - Carboxylate Degradation

$\beta$-D-glucuronide and D-glucuronate degradation $\quad X$

2-amino-3-carboxymuconate semialdehyde degradation to 2-oxopentenoate $\mathrm{X}$

2-methylcitrate cycle I $\quad X$

Degradation/Utilization/Assimilation - Chlorinated Compound Degradation

2-chlorobenzoate degradation

4,5-dichlorocatechol degradation

$X$

chlorinated phenols degradation

pentachlorophenol degradation

Degradation/Utilization/Assimilation - Degradation/Utilization/Assimilation - Other

acrylonitrile degradation I

octane oxidation

Degradation/Utilization/Assimilation - Fatty Acid and Lipid Degradation

cholesterol degradation to androstenedione I (cholesterol oxidase) phospholipases

triacylglycerol degradation

Degradation/Utilization/Assimilation - Inorganic Nutrient Metabolism

aminomethylphosphonate degradation

$X$

cyanate degradation

$X$

dimethyl sulfide degradation II (oxidation)

X $\quad X$

$X$

$\mathrm{X}$

$X$

$X$ 


\section{Pathway Class}

glyphosate degradation II

nitrate reduction $\mathrm{V}$ (assimilatory)

$x$

nitrogen fixation I (ferredoxin)

$x$

thiosulfate disproportionation IV (rhodanese)

$x$

Degradation/Utilization/Assimilation - Nucleoside and Nucleotide Degradation

guanosine nucleotides degradation III

pyrimidine deoxyribonucleosides degradation

UTP and CTP dephosphorylation I

Degradation/Utilization/Assimilation - Polymeric Compound Degradation

alginate degradation

$\mathrm{X}$

\section{Degradation/Utilization/Assimilation - Secondary Metabolite Degradation}

$\beta$-D-glucuronide and D-glucuronate degradation

$\mathrm{X}$

$\mathrm{N}$-acetylglucosamine degradation I

$\mathrm{X}$

D-sorbitol degradation I

limonene degradation I (D-limonene)

$X$

limonene degradation II (L-limonene)

$\mathrm{X}$

\section{Generation of Precursor Metabolite and Energy}

aerobic respiration I (cytochrome c)

$x$

glycerol-3-phosphate to cytochrome bo oxidase electron transfer

$\mathrm{X}$

glycerol-3-phosphate to fumarate electron transfer

$\mathrm{X}$

mixed acid fermentation

$\mathrm{X}$

NADH to cytochrome bd oxidase electron transfer I

$\mathrm{X}$

NADH to cytochrome bo oxidase electron transfer I

$\mathrm{X}$

pentose phosphate pathway (oxidative branch) I

$\mathrm{X}$

proline to cytochrome bo oxidase electron transfer

$x$

TCA cycle I (prokaryotic)

TCA cycle IV (2-oxoglutarate decarboxylase)

$x$ 


\section{Exceptional cases of Gynmostomma and Ceanothus}

Although, there were more than 200 shared metabolic pathways between C-I and C-III Frankia (discussed earlier), a total of 67 metabolic pathways were detected which were present in the C-III group but not in CI. Furthermore, the nif cluster of C-III also showed a distinct distinction between them and C-I, i.e. nifV gene was located at distance from other nif genes in C-III, whereas. in C-I all the nif genes were clustered together. The same finding was also reported earlier (Normand et al. 2007). All these factors may cumulatively affect the infection cycle of C-III Frankia in Gynmostomma (the single Casurinaceae family that cannot be infected by $\mathrm{C}-\mathrm{I}$ but by $\mathrm{C}$-III). Since symbiosis also depends on the signaling machinery between the host plant and Frankia, we compared the signal peptide proteins present in C-I and C-III groups (Fig. 2). A total of 59 different types of signal proteins were found in the C-Ill group that was absent in C-I, which contain 115 types of signal peptide proteins that are absent in C-II or C-III. This demonstrated the fact that the signaling mechanisms adopted by these different groups of Frankia are quite distinct. Similarly, we have compared the signal peptides of Cll and CIII groups of Frankia and got a set of 80 unique signalP proteins for the C-II group. Those were absent in the C-III and C-I groups indicating a discrepancy of signaling cascade between these two groups. We have not considered the atypical C-IV Frankia since they always remain ineffective irrespective of their host plant.

Root nodule formation doesn't only depend upon the relative abundance of Frankia in rhizospheric soil but also, commodiously hinges on the acceptability of the host plant towards a specific type of Frankia (Tekaya et al. 2018). Since our knowledge regarding the Gynmostomma as well as Ceanthous genomes and their metabolism along with the signaling system is very elusive, further contemplation especially investigation on root extract of such plants is required to reveal this aforementioned unusual behavior of Frankia nodulation.

\section{Intra-cluster mutualism among Frankia}

The complementation index among different clusters of Frankia revealed higher intra-cluster mutual-ism than inter-cluster mutualism. The value ranged from 0.58-0.96 (for $\mathrm{Cl}$ ), 0.70-0.89(for $\mathrm{Cll}$ ), 0.67-0.99(for CIII), 0.62-0.96(C-IV). Although Ben et al. (2018) proposed that the diversity of Frankia in their host plant root nodules is independent of the Frankia diversity and abundance but is dependent on the choice of the host plant, the intra-cluster complementation may support the host-specific nature of Frankia in the majority of cases. However, further future studies will strengthen this proposal.

\section{Conclusion}

There are two major hypotheses regarding the evolution of Actinorhizal plants. One of them deals with the selective advantage of widely diverged plant genera to associate with Frankia in some specialized ecological niche (24). The second hypothesis, on contrary, takes us to the early cretaceous period when the Earth's atmosphere lacked nitrogen. Due to the inadequacy of nitrogen, the woody actinorhizal plants were amassed to make the symbiotic association with N2 fixing Frankia (Normand P, Bouquet, 1989). Subsequently, with the evolution of Earth, nitrogen becomes more attainable and the power of selective 
advantages decreased. This happened around $100 \mathrm{M}$ years ago. As a result, some of the contemporary plant families lost their ability to make an association with Frankia. However, in places where nitrogen is still unavailable or present in very low concentration (in case of landslide and soil erosion), the ancestral property for maintaining a mutualistic association between actinorhizal plants and Frankia remains unchanged as a result of selective advantage. Phylogenetic study based on the nifH gene (Normand $\mathrm{P}$, Bouquet, 1989) along with DNA hybridization studies (Bousquet et al. 1989) fully supported the second hypothesis mentioning the importance of $\mathrm{N}_{2}$ availability as the major force of selective advantage for a symbiotic alliance of host-Frankia interaction. Moreover, soil-dwelling bacteria especially those living in a mutualistic association with specific host plants are believed to encounter two different levels of selection pressure. The first selection happens during successful adaptation in soil and the second one occurs when the bacteria start the symbiotic connotation with their respective host. Previous studies have reported the genomic expansion as well as reductions among facultative symbionts as their strategic response to the new environment as well as to successive symbiotic signals. Frankia being nitrogenfixing actinobacteria is vital from both ecological and agricultural aspects. Different molecular biologybased analyses along with several in-silico studies have exposed the genomic diversity present among the members of this genus. However, the ecological aspect of Frankia was not much ex-plored. In this study, we have specifically focused on the ecological principles acting on this genus which provide them the ability of host specificity or host differentiation. We have used the reverse ecology analysis among 44 Frankia strains distributed in 4 clusters. The analysis revealed C-I Frankia as a general competitor against other clusters and validated their host specificity towards the Hamamelidae subclass of actinorhizal plants. Another very exciting finding was the presence of niche partitioning canon between C-II and C-IV Frankia strains that allow them to share the same nodule via utilizing different nutrition sources especially for carbohydrate and aromatic amino acids. In this study we have also focused on the nonFrankia genera those were reported to be found within the same root nodule where Frankia inhabit. In ecosystem, the possibility of co-existance relies upon two factors- competition and complementation. We used reverse ecology algorithm to assess the interaction among Frankia and other co-inhabiting strains. We found these co-inhabiting strains are actually supporting strains helping in Frankia lifestyle irrespective of their different clustering pattern. Probably, these supporting strains are helpful for the host plants too. However, to prove this hypothesis we will need complete genome sequences for all the host plants as well as their metabolic profiling. We hope with advanced NGS techniques we will be able to get all the necessary information for the host plants and that will definitely reveal a more complex and interesting information regarding the co-existance of Frankia, their supporting strains and host plants.

\section{Declarations}

\section{Acknowledgments}

We acknowledge S Das and C Sakar for initially standardizing the software. We also acknowledge the Biswa Bangla Genome Center and University of North Bengal for supporting this work.

\section{Author contributions}

Page $14 / 19$ 
AS and MG conceived and developed the idea. IS, GS \& AS designed the experiment and did the bioinformatic work. SB, IS and AS contributed in analyzing the data and wrote the final draft of the manuscript. All authors have read and approved the manuscript.

\section{Conflict of interest}

The authors declare that the research paper was written in the absence of any commercial or financial relationships that could be construed as the real or potential conflict of interest. Thus authors declare no conflict of interest.

\section{Animal Issues}

No animal or human was treated as the sample in this work.

\section{References}

1. Allaire, J., (2012). RStudio: integrated development environment for R. Boston, MA, 770, p.394.

2. Amann RI, Ludwig W, Schleifer KH. (1995) Phylogenetic identification and in situ detection of individual microbial cells without cultivation. Microbiol Rev. 59:143-69.

3. Barabasi A, Albert R. 1999. Emergence of scaling in random networks. Science, 286: 509-512

4. Barabasi AL. 2009. Scale-free networks: a decade and beyond. Science, 325: 412-413

5. Ben Tekaya, S., Guerra, T., Rodriguez, D., Dawson, J.O. and Hahn, D., (2018). Frankia diversity in host plant root nodules is independent of abundance or relative diversity of Frankia populations in corresponding rhizosphere soils. Applied and environmental microbiology, 84(5), pp.e02248-17.

6. Benson, D.R. and Dawson, J.O., (2007). Recent advances in the biogeography and genecology of symbiotic Frankia and its host plants. PhysiologiaPlantarum, 130(3), pp.318-330.

7. Benson, D.R., Vanden Heuvel, B.D. and Potter, D., (2004). Actinorhizal symbioses: diversity and biogeography. Plant microbiology, pp.97-127.

8. Berlow, E. L., Dunne, J. A., Martinez, N. D., Stark, P. B., Williams, R. J., \& Brose, U. (2009). Simple prediction of interaction strengths in complex food webs. Proceedings of the National Academy of Sciences, 106(1), 187-191.

9. Berry, A.M., Mendoza-Herrera, A., Guo, Y.Y., Hayashi, J., Persson, T., Barabote, R., Dem-chenko, K., Zhang, S. and Pawlowski, K., (2011). New perspectives on nodule nitrogen assimilation in actinorhizal symbioses. Functional Plant Biology, 38(9), pp.645-652.

10. Berry, D. and Widder, S., 2014. Deciphering microbial interactions and detecting keystone species with co-occurrence networks. Frontiers in microbiology, 5, p.219.

11. Borenstein $\mathrm{E}$, Kupiec M, Feldman MW, et al. Large-scale reconstruction and phylogenetic analysis of metabolic environments. ProcNatlAcad Sci. 2008; 105:14482-7.

12. Bousquet J, Girouard E, Strobeck C, Dancik BP, Lalonde M (1989). Restriction fragment polymorphisms in the rDNA region among seven species of AlnusandBetulapapyrifera. Plant and soil. 
118(1-2):231-40.

13. Cao, Y., Wang, Y., Zheng, X., Li, F., \& Bo, X. (2016). RevEcoR: an R package for the reverse ecology analysis of microbiomes. BMC bioinformatics, 17(1), 1-6.

14. Cao, Yang, Yuanyuan Wang, Xiaofei Zheng, Fei Li, and Xiaochen Bo. (2016). "RevEcoR: an R package for the reverse ecology analysis of microbiomes." BMC bioinformatics 17, no. 1: 1-6.

15. Carr R, Borenstein E (2012). NetSeed: a network-based reverse-ecology tool for calculating the metabolic interface of an organism with its environment. Bioinforma Oxf Engl ;28:734-5

16. Caspi, R., Foerster, H., Fulcher, C.A., Kaipa, P., Krummenacker, M., Latendresse, M., Paley, S., Rhee, S.Y., Shearer, A.G., Tissier, C. and Walk, T.C., (2007). The MetaCyc Database of metabolic pathways and enzymes and the BioCyc collection of Pathway/Genome Databases. Nucleic acids research, 36: pp.D623-D631.

17. Cissoko, M., Hocher, V., Gherbi, H., Gully, D., Carré-Mlouka, A., Sane, S. \& Fournier, P. (2018). Actinorhizal signaling molecules: Frankia root hair deforming factor shares properties with NIN inducing factor. Frontiers in plant science, 9, 1494.

18. Cronquist, A., (1968). The evolution and classification of flowering plants. The evolution and classification of flowering plants.

19. Duffy, J. E., Cardinale, B. J., France, K. E., McIntyre, P. B., Thébault, E., \& Loreau, M. (2007). The functional role of biodiversity in ecosystems: incorporating trophic complexity. Ecology letters, 10(6), 522-538.

20. Dunne, J. A. (2006). The network structure of food webs. Ecological networks: linking structure to dynamics in food webs, 27-86.

21. Dunne, J. A., Williams, R. J., \& Martinez, N. D. (2002). Network structure and biodiversity loss in food webs: robustness increases with connectance. Ecology letters, 5(4), 558-567.

22. Faust, K., \& Raes, J. (2012). Microbial interactions: From networks to models. Nature Reviews Microbiology, 10, 538- 550.

23. Finke, D.L. and Snyder, W.E., (2008). Niche partitioning increases resource exploitation by diverse communities. Science, 321(5895), pp.1488-1490.

24. Friedman, J., \& Alm, E. J. (2012). Inferring correlation networks from genomic survey data. PLoS Computational Biology, 8, e1002687.

25. Gilbert, N., Russo, G., Marris, E., Lane, N., Sukhdev, P., Turner, W. R., ... \& Wilcove, D. S. (2009). The entangled bank unravels.

26. Gtari, M., Brusetti, L., Skander, G., Mora, D., Boudabous, A. and Daffonchio, D., (2006). Isolation of Elaeagnus-compatible Frankia from soils collected in Tunisia. FEMS microbiology letters, 234(2), pp.349-355.

27. Hugenholtz, P., Goebel, B. M., \& Pace, N. R. (1998). Impact of culture-independent studies on the emerging phylogenetic view of bacterial diversity. Journal of bacteriology, 180(18), 4765-4774. 
28. Ings, T. C., Montoya, J. M., Bascompte, J., Blüthgen, N., Brown, L., Dormann, C. F., ... \& Woodward, G. (2009). Ecological networks-beyond food webs. Journal of animal ecology, 78(1), 253-269.

29. Kanehisa, M., (2002). The KEGG database. In Novartis Foundation Symposium (pp. 91 100).Chichester; New York; John Wiley; 1999.

30. Kéfi, S., Berlow, E. L., Wieters, E. A., Navarrete, S. A., Petchey, O. L., Wood, S. A., ... \& Brose, U. (2012). More than a meal... integrating non-feeding interactions into food webs. Ecology letters, 15(4), 291300 .

31. Lassalle, F., Muller, D., \&Nesme, X. (2015). Ecological speciation in bacteria: reverse ecology approaches reveal the adaptive part of bacterial cladogenesis. Research in microbiology, 166(10), 729-741.

32. Lefort, V., Desper, R. and Gascuel, O., (2015). FastME 2.0: a comprehensive, accurate, and fast distance-based phylogeny inference program. Molecular biology and evolution, 32(10), pp.27982800 .

33. Levy R, Borenstein E 2012. Reverse Ecology: from systems to environments and back. AdvExp Med Biol. 751:329-345.

34. Levy R, Carr R, Kreimer A, et al. (2015) NetCooperate: a network-based tool for inferring host-microbe and microbe-microbe cooperation. BMC Bioinf.; 16:164.

35. Mansour, S., Swanson, E., McNutt, Z., Pesce, C., Harrington, K., Abebe-Alele, F., Simpson, S., Morris, K., Thomas, W.K. and Tisa, L.S., (2017). Permanent draft genome sequence for Frankia sp. strain Ccl49, a nitrogen-fixing bacterium isolated from Casuarina cunninghamiana that infects Elaeagnaceae. Journal of genomics, 5, p.119.

36. Meier-Kolthoff, J.P. and Göker, M., (2019). TYGS is an automated high-throughput platform for stateof-the-art genome-based taxonomy. Nature communications, 10(1), pp.1-10.

37. Meier-Kolthoff, J.P., Auch, A.F., Klenk, H.P. and Göker, M., (2013). Genome sequence-based species delimitation with confidence intervals and improved distance functions. BMC bioin-formatics, 14(1), pp.1-14.

38. Moriya, Y., Itoh, M., Okuda, S. and Kanehisa, M., (2005). KAAS: KEGG automatic annotation server. Genome Informatics, 5, p.2005.

39. Ngom, M., Oshone, R., Hurst IV, S.G., Abebe-Akele, F., Simpson, S., Morris, K., Sy, M.O., Champion, A., Thomas, W.K. and Tisa, L.S., (2016). Permanent draft genome sequence for Frankia sp. strain CeD, a nitrogen-fixing actinobacterium isolated from the root nodules of Casuarina equistifolia grown in Senegal. Genome announcements, 4(2), pp.e00265-16.

40. Nnelson, L.M. and Edie, S.A., (1991). Nodule carbohydrate composition and nitrogen fixation in pea (Pisumsativum L.): Effect of Rhizobium strain and NH4NO3. Soil Biology and Biochemistry, 23(7), pp.681-688.

41. Normand P, Bouquet J. (1989) Phylogeny of nitrogenase sequences in Frankia and other nitrogenfixing microorganisms. Journal of molecular evolution. 29(5):436-47. 
42. Normand, P., Lapierre, P., Tisa, L. S., Gogarten, J. P., Alloisio, N., Bagnarol, E., ... \& Benson, D. R. (2007). Genome characteristics of facultatively symbiotic Frankia sp. strains reflect host range and host plant biogeography. Genome research, 17(1), 7-15.

43. Nouioui, I., Gtari, M., Göker, M., Ghodhbane-Gtari, F., Tisa, L.S., Fernandez, M.P., Normand, P., Huntemann, M., Clum, A., Pillay, M. and Varghese, N., (2016). Draft genome sequence of Frankia strain G2, a nitrogen-fixing actinobacterium isolated from Casuarina equisetifolia and able to nodulate actinorhizal plants of the order Rhamnales. Genome announcements, 4(3), pp.e00437-16.

44. Pedraza, R.O., Ramírez-Mata, A., Xiqui, M.L. and Baca, B.E., (2004). Aromatic amino acid aminotransferase activity and indole-3-acetic acid production by associative nitrogen-fixing bacteria.FEMS microbiology letters, 233(1), pp.15-21.

45. Poisot, T., Stouffer, D. B., \& Gravel, D. (2015). Beyond species: why ecological interaction networks vary through space and time. Oikos, 124(3), 243-251.

46. Pujic, P., Bolotin, A., Fournier, P., Sorokin, A., Lapidus, A., Richau, K.H., Briolay, J., Mebarki, F., Normand, P. and Sellstedt, A., (2015). Genome sequence of the atypical symbiotic Frankia R43 strain, a nitrogen-fixing and hydrogen-producing actinobacterium. Genome announcements, 3(6), pp.e0138715.

47. Ramírez-Saad H, Janse JD, Akkermans AD. (1998). Root nodules of Ceanothus caeruleus contain both the N2-fixing Frankia endophyte and a phylogetically related Nod-/Fix-actinomycete. Canadian journal of microbiology.44(2):140-8.

48. Schwencke, J. and Carú, M., (2001). Advances in actinorhizal symbiosis: host plant-Frankia interactions, biology, and applications in arid land reclamation. A review.Arid Land Research and Management, 15(4), pp.285-327.

49. Schwintzer, C.R., (2012). The biology of Frankia and actinorhizal plants.Academic Press, Inc

50. Sellstedt, A. and Richau, K.H., (2013). Aspects of nitrogen-fixing Actinobacteria, in particular freeliving and symbiotic Frankia. FEMS Microbiology Letters,342(2), pp.179-186

51. Sen, A., Daubin, V., Abrouk, D., Gifford, I., Berry, A.M. and Normand, P., (2014). Phylogeny of the class Actinobacteria revisited in the light of complete genomes. The orders 'Frankiales' and Micrococcales should be split into coherent entities: proposal of Frankiales ord. nov., Geodermatophilales ord. nov., Acidothermales ord. nov.andNakamurellales ord. nov.International journal of systematic and evolutionary microbiology, 64(11), pp.3821-3832.

52. Simonet, P., Haurat, J., Normand, P., Bardin, R. \& Moiroud, A. (1986). Localization of nif genes on a large plasmid in Frankia sp. strain ULQ0132105009. Mol Gen Genet 204, 492-495.

53. Suzuki, K., Yoshida, K., Nakanishi, Y. and Fukuda, S., 2017. An equation-free method reveals the ecological interaction networks within complex microbial ecosystems. Methods in Ecology and Evolution, 8(12), pp.1774-1785.

54. Tekaya, S.B., Guerra, T., Rodriguez, D., Dawson, J.O. and Hahn, D., (2018). Frankia diversity in host plant root nodules is independent of abundance or relative diversity of Frankia populations in corresponding rhizosphere soils. Applied and environmental microbiology, 84(5). 
55. Vemulapally, S., Guerra, T. and Hahn, D., (2019). Localization of typical and atypical Frankia isolates from Casuarina sp. in nodules formed on Casuarina equisetifolia. Plant and Soil, 435(1), pp.385-393.

56. Zhang, W. (2011). Constructing ecological interaction networks by correlation analysis: hints from community sampling. Network Biology, 1(2), 81.

\section{Figures}

\section{Figure 1}

Unique and shared transporter proteins among four clusters of Frankia. The Venn diadram was generated in $\mathrm{R}$.

\section{Figure 2}

Venn diagram of unique and shared signal peptides among C-I, II and III of Frankia. The Venn diagram was generated in R. We have not considered the atypical C-IV Frankia since they always remain ineffective irrespective of their host plant.

\section{Supplementary Files}

This is a list of supplementary files associated with this preprint. Click to download.

- Supplementaryfigure1.tif

- Supplementaryfigure2.pdf

- Supplementaryfigure3.pdf

- Supplementaryfile1.xlsx

- Supplementaryfile2.xlsx

- Supplementarytable1.docx 